\title{
TANG DYNASTY CHAIR FEATURE DESIGN BASED ON KANSEI EVALUATION AND EYE TRACKING SYSTEM
}

\author{
Zhonghua Zhagn, Boming Xu \\ College of Furnishings and Industrial Design \\ Nanjing Forestry University \\ Nanjing, China \\ (Received March 20I9)
}

\begin{abstract}
Tang dynasty (AD 618-907) chairs were manufactured during an important period of Chinese furniture development. This paper aims to identify design elements that impact on people's subjective impressions, so as to guide the design of Tang dynasty style chairs. The study combined eye tracking and Kansei evaluation methods to assess the semantic reception of Tang dynasty chairs. The results showed that the influential factors can be grouped into two main categories: decoration and shape. The decorative features of Tang dynasty chairs that have the most significant impact on visual attention were identified. The study determined that the backrests, armrests, and legs of Tang dynasty chairs design were the most important morphological features. Through these morphological features, we can define the Tang dynasty chair style, guide the design of modern Tang dynasty style chairs, and carry out targeted design of Tang dynasty chair style features.
\end{abstract}

KEYWORD: Kansei evaluation, eye tracking, Tang dynasty, chair.

\section{INTRODUCTION}

The Tang dynasty was a prosperous period of China's history during which it was predominantly a feudal society. The stylized form and elegant decoration of Tang dynasty furniture are material manifestations of the splendid culture (Zhu 2016, Lee et al. 2018) and aesthetics of the Tang dynasty, and pieces dating from the period which has significant research value when studying the developmental history of traditional Chinese furniture. During the Tang dynasty, high and low furniture coexisted and there was a considerable variety of forms produced. At present, research on Tang dynasty chairs is mainly conducted from the historical perspective (Zanous and Sangari 2018, Ying 2018) and using traditional craftwork analysis (Wang et al. 2015, Hu et al. 2019a). No in-depth study of the emotional impact of Tang dynasty chairs on people who view or use them has been carried out. With the development of modern society, 
people have higher requirements regarding furniture styles and individual tastes (Wan et al. 2018). Tang dynasty chairs have unique styles and characteristics (Hu et al. 2019b, 2019c), but the style has not been widely developed. The reason is that Tang dynasty chair design features are not obvious, and at the same time, the subjective feelings of people regarding Tang dynasty chairs have been ignored. As a result, the general aim of this study is to identify design elements that impact on people's subjective impressions, so as to guide the design of Tang dynasty style chairs. In the majority of studies investigating the relationships between form and eye movement (Loyola et al. 2015, Zhou et al. 2014), Kansei evaluations and eye movement analyses have been carried out separately (Guo et al. 2016, Hsu et al. 2017, Vieira et al. 2017, Wang et al. 2016), so it is difficult to identify exactly which features of the samples affected people's Kansei evaluations. The present study used an eye tracking system to record the eye movements of subjects while they performed Kansei evaluations of Tang dynasty chairs. Eye tracking systems can accurately measure the eye fixation points during subjective evaluations (Khalighy et al. 2015), so as to determine which of the Tang dynasty chair features played the principal role influencing the subjective evaluations.

The Tang dynasty had a history lasting more than a thousand years (Wang et al. 2015). At that time, the materials used to make chairs were predominantly woods (Dong et al. 2017), which are not easy to store or preserve. There are, therefore, only a few examples of Tang dynasty chairs now in existence. Samples in this research are reconstructed based on the shape of the chairs depicted in paintings, the proportions of the chairs compared to people in the paintings, information regarding the size of the surviving Tang dynasty chairs, and those recorded in ancient literature. With these sources and the size of other cultural relics, the proportions and forms of Tang dynasty chairs were estimated. Finally, using the principle of two-point perspective, the Tang dynasty chairs were then depicted by line drawings. Through the above process, the style of Tang dynasty chairs was clearly represented, and this work, therefore, plays an important role in the restoration and cultural appreciation of Tang dynasty chairs.

\section{MATERIALS AND METHODS}

\section{Stimuli}

The first step in a Kansei evaluation is to gather as many relevant words and expressions as possible for the definition of the semantic scope of the study (Trujillo et al. 2016, Castilla et al. 2017). The majority of the words selected were adjectives used to describe Tang dynasty chairs and the Tang dynasty style. In the present research, the majority of the words were found in documents on the Internet, in newspapers, journals, scientific publications, and professional magazines. During this phase, a total of 140 expressions were collected. There were too many adjectives to be used in the Kansei questionnaire, and most of the words were repeated or similar, so further selection was required. A group including two professors, two design experts, and two students were organized to analyze and reduce the semantic adjectives down to an appropriate number. Finally, only 8 pairs of adjectives were preserved: (a) plain-luxury, (b) flowing-stable, (c) disordered-harmonious, (d) simple-intricate, (e) round-straight, (f) delicate- robust, (g) stockyexquisite, and (h) slim-thick.

In this study, images of the chairs on objects, in paintings, and in murals were collected and classified. Incomplete, unclear, or ambiguous depictions were then excluded, leaving a total of 11 pieces in the study group (Fig. 1). In order to emphasize sample morphology, it was necessary to exclude variations in color, material, light, and shadow from the sample images to avoid visually misleading subjects. The samples were, therefore, all presented in the form of line drawings 
and observed at a $45^{\circ}$ angle. The present study concluded with using 11 chairs as stimuli and 8 pairs of Kansei words as measurement scales for the following semantic differential (SD) evaluation and eye tracking experiment.

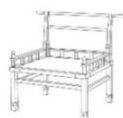

1

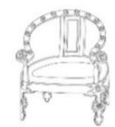

5

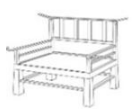

9

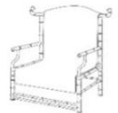

2

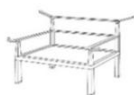

6

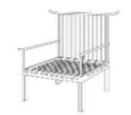

10
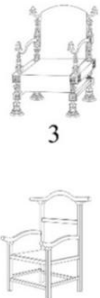

7

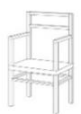

11

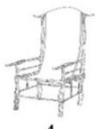

4

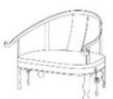

8

Fig. 1: Experimental stimuli.

\section{Subjects}

In the current study, subjects required a certain cognitive and sensory sensitivity to morphology. Many scholars have studied (Winston and Cupchik 1992, Nodine et al. 1993) that formal art training influences perception and reception. The responses of people trained in artistic methods or theory differ from controls in their perception of form. Most of the subjects chosen for the experiment were experienced practitioners in a relevant field. The average age of the subjects was 28 years (Tab. 1). All of the subjects had normal or corrected-to-normal vision. They all signed written consent before the experiment and received a gift as a reward afterwards.

Tab. 1: Subject structure.

\begin{tabular}{|l|l|c|c|}
\hline Subject structure & N & $\mathbf{\%}$ \\
\hline \multirow{3}{*}{ Gender } & Male & 10 & 50 \\
\cline { 2 - 4 } & Female & 10 & 50 \\
\hline \multirow{2}{*}{ Age } & Up to 30 years & 12 & 60 \\
\cline { 2 - 4 } & Above 30 years & 8 & 40 \\
\hline \multirow{2}{*}{ Education } & University education & 11 & 55 \\
\cline { 2 - 4 } & Non-university education & 9 & 45 \\
\hline \multirow{2}{*}{ Working status } & Furniture industry practitioners & 18 & 90 \\
\cline { 2 - 4 } & Non-furniture industry practitioners & 2 & 10 \\
\hline
\end{tabular}

\section{Procedure}

The experiment was carried out at Nanjing Forestry University in China. The environment of the laboratory was quiet in order to eliminate external interference, and the lighting conditions were stable. A TOBII eye tracker was used in the experiment (Fig. 2). 


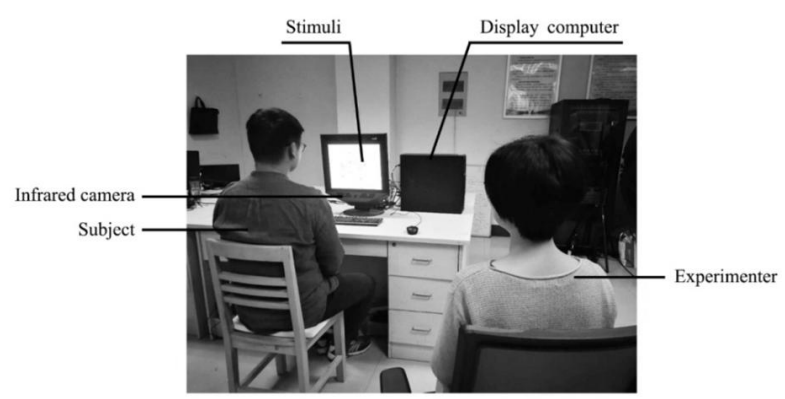

Fig. 2: Experimental environment and equipment.

Eye movement data was recorded using a Clearview 2.7.0 software system. In order to make the experimental results more accurate and meaningful, it was necessary to pre-define the areas of interest (AOIs) in the images. Each of the Kansei words was explained to allow the participants to fully understand intended meanings. In order to allow subjects to better understand the samples, each participant was required to experience a brief introduction to the style and characteristics of Tang dynasty chairs whose specific information or names were not introduced. Before the experiment, participants were also given instructions regarding the test procedures and underwent an experiment trial so that they would be familiar with the task requirements and methodology in advance.

During the experiment, subjects were asked to carry out a Kansei evaluation of each chair and evaluate their reception according to a 7-point-Likert scale, based on the stimuli that appeared on the screen. In order to avoid subjects' fixation points moving outside the screen, the results of perceptual evaluations were dictated by the subjects, and the experimenter recorded the scores for the subjects. In order to keep the subjects focused during the experiment and to avoid visual fatigue, computers or mobile phones could not be used for two hours before the experiment was carried out, and a rest was taken every ten minutes during the experiment. The experimental process is shown in Fig. 3.

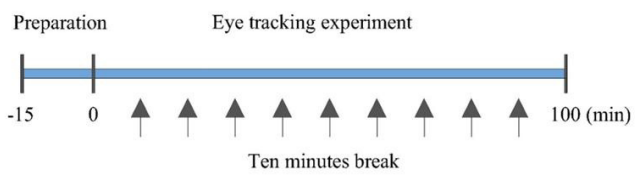

Fig. 3: Experimental flow chart.

When the experiment started, subjects observed the first pair of perceptual vocabulary scales (5s), the gaze concentration point (2s), then the chair stimuli (7s), and finally, the first pair of perceptual vocabulary scales again $(5 \mathrm{~s})$. The subjects then dictated their scores. Then they observed the second of the semantic vocabulary pairs and repeated the process, until the last chair and the last vocabulary scale was dictated, and the experiment ended (Fig. 4). The research flowchart is shown in Fig. 5. 

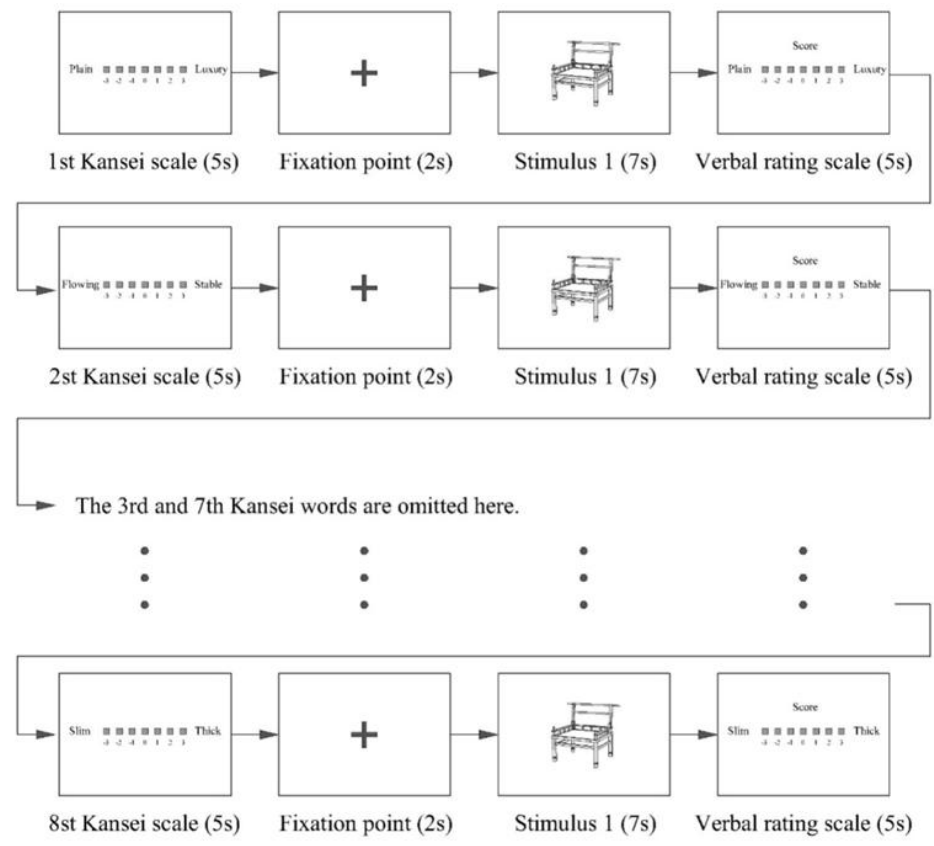

Fig. 4: Experimental procedure for stimulus 1.

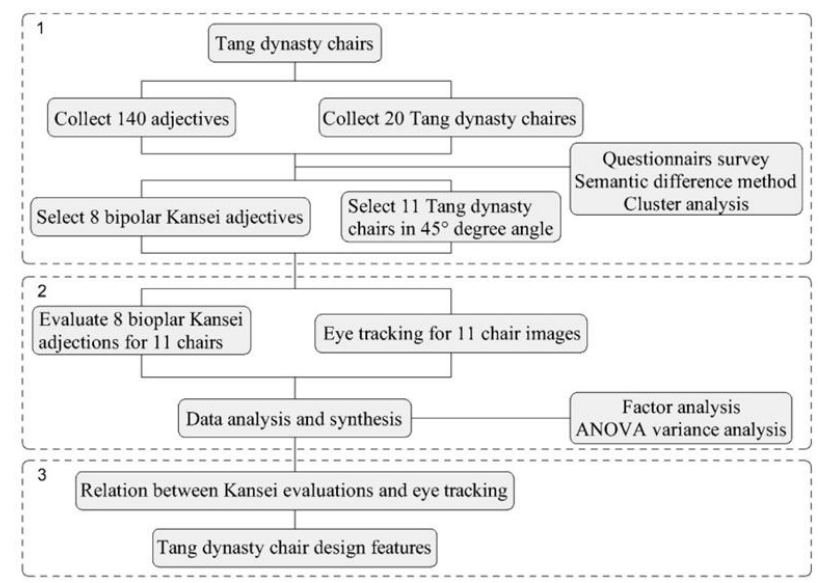

Fig. 5: Flowchart.

\section{Data processing}

Data were processed statistically using Statistical Product and Service Solutions (SPSS22) software. First, factor analysis was used to obtain the information about the general impressions eople experienced in response to the Tang dynasty. The second stage of the analysis consisted of an ANOVA variance analysis to analyze the relationship between the Kansei evaluation and eye movements. The significance level was set at $\mathrm{p}<0.05$. 


\section{RESULTS}

\section{Factor analysis for the Kansei evaluation}

The results the Tang dynasty evaluation are shown in Fig. 6. It can be seen from the subjective evaluations that the profiles produced vary in response to the different stimuli.

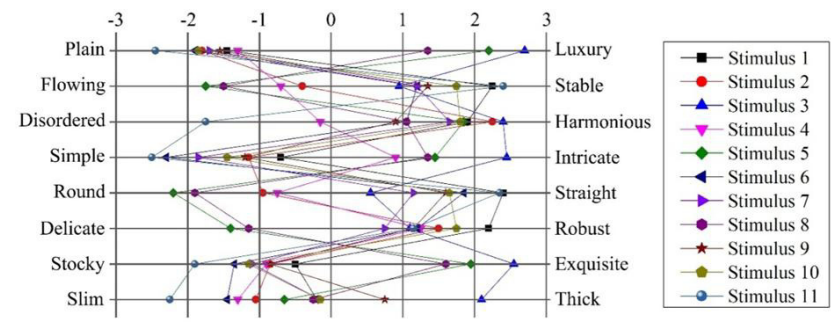

Fig. 6: Evaluation results Tang dynasty chairs.

Factor analysis was conducted to extract the factors from the SD evaluation data. According to factor extraction conventions, the eigen value must be larger than one. Two factors were generalized from the eight Kansei word pairs, and factor rotation was applied with the varimax method (Tab. 2).

Tab. 2: Factor analysis of Kansei evaluation.

\begin{tabular}{|l|c|c|c|}
\hline \multicolumn{1}{|c|}{ Bipolar adjectives } & Factor 1 & Factor 2 & Communality \\
\hline Round-Straight & 0.944 & 0.094 & 0.898 \\
\hline Delicate-Robust & 0.890 & 0.025 & 0.593 \\
\hline Plain-Luxury & 0.681 & 0.671 & 0.843 \\
\hline Simple-Intricate & -0.678 & -0.619 & 0.900 \\
\hline Slim-Thick & 0.077 & -0.947 & 0.793 \\
\hline Disordered-Harmonious & 0.035 & 0.769 & 0.942 \\
\hline Stocky-Exquisite & 0.651 & 0.720 & 0.903 \\
\hline Variance (\%) & 49.191 & 35.639 & \\
\cline { 1 - 2 } Cumulative variance (\%) & 49.191 & 84.830 & \\
\cline { 1 - 2 } & & \multicolumn{2}{|l}{} \\
\cline { 1 - 2 }
\end{tabular}

Factor 1 consisted of flowing-stable, round-straight, delicate-robust, plain-luxury, and simple-intricate. Factor 2 included slim-thick, disordered-harmonious, and stocky-exquisite. Factor 1 explained $49.2 \%$ of the variance and Factor 2 explained 35.6\% (the cumulative explained variance equaled 84.8\%). The adjectives in Factor 1 (e.g. round-straight, simple-intricate) represented aspects of shape, whereas those in Factor 2 (e.g. slim-thick, disordered-harmonious) represented decoration.

\section{Spanning the Kansei space based on responses to the stimuli}

The dimensions of the Kansei space were established based on subjects' responses to the stimuli through the factor analysis. Shape was assigned to the $\mathrm{X}$-axis, with shape-flowing and shape-stable as the two extreme values. Decoration was assigned to the $\mathrm{Y}$-axis, with decorationdisordered and decoration-harmonious as the two extreme values. 
Tab. 3: The factor scores for the Tang dynasty backrest armchair stimuli.

\begin{tabular}{|c|c|c|}
\hline Sample & Factor 1 & Factor 2 \\
\hline 1 & 1.14 & 0.57 \\
\hline 2 & -0.14 & -0.31 \\
\hline 3 & 0.06 & 2.29 \\
\hline 4 & -0.65 & -0.86 \\
\hline 5 & -1.87 & 0.21 \\
\hline 6 & 0.51 & -0.67 \\
\hline 7 & 0.48 & -0.04 \\
\hline 8 & -1.65 & 0.07 \\
\hline 9 & 0.68 & 0.28 \\
\hline 10 & 0.91 & 0.21 \\
\hline 11 & 0.52 & -1.73 \\
\hline
\end{tabular}

The sample factor score matrix was then extracted (Tab. 3), delineating the distribution of the stimuli in the Kansei space (Fig. 7).

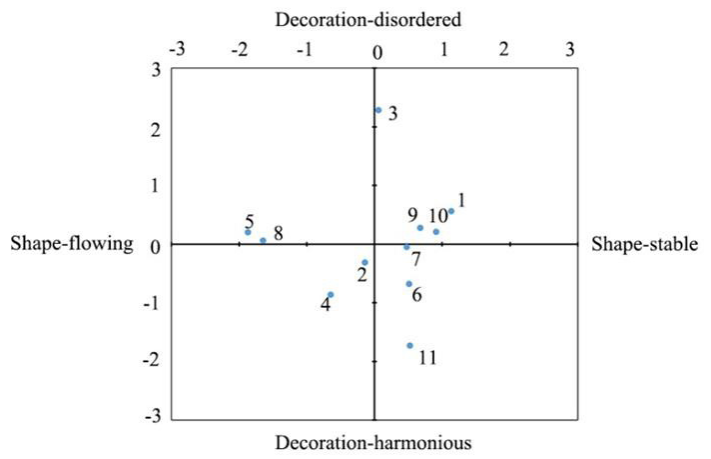

Fig. 7: Distribution of the stimuli in the Kansei space.

\section{Analysis of eye-tracking outcomes}

In order to understand whether there was a significant difference in the number of fixation points between the four quadrants and whether the two factors had an effect on the fixation points, a two-way ANOVA analysis was used for data analysis and processing. Tab. 4 shows the average fixation points and fixation time for the stimuli.

Tab. 4: The average fixation points and fixation time for the stimuli.

\begin{tabular}{|c|c|c|}
\hline Stimuli & Fixation points (number) & Fixation time (s) \\
\hline 1 & 16.06 & 3.56 \\
\hline 2 & 14.43 & 3.06 \\
\hline 3 & 16.51 & 3.44 \\
\hline 4 & 13.97 & 2.94 \\
\hline 5 & 16.76 & 3.17 \\
\hline 6 & 14.03 & 3.09 \\
\hline
\end{tabular}




\begin{tabular}{|c|c|c|}
\hline 7 & 14.75 & 3.60 \\
\hline 8 & 16.28 & 3.36 \\
\hline 9 & 15.98 & 3.13 \\
\hline 10 & 15.76 & 3.24 \\
\hline 11 & 12.79 & 2.86 \\
\hline
\end{tabular}

Tab. 5 shows the results for the two-way ANOVA analysis of fixation points. The effect of interaction between the two factors on the number of fixation points was insignificant $(p>0.05)$. In addition, there was no significant correlation between shape-stable and the number of fixation points $(p>0.05)$. There was, however, a significant correlation between decoration-disordered and the number of fixation points.

Tab. 5: 2-way ANOVA of fixation points.

\begin{tabular}{|l|c|c|c|c|c|}
\hline \multicolumn{1}{|c|}{ Source } & $\begin{array}{c}\text { type III Sum } \\
\text { of squares }\end{array}$ & df & Mean square & F & sig \\
\hline Shape-stable & 0.390 & 1 & 0.390 & 1.099 & 0.329 \\
\hline Decoration-disordered & 13.023 & 1 & 13.023 & 36.690 & 0.001 \\
\hline Shape-stable * decoration-disordered & 0.006 & 1 & 0.006 & 0.017 & 0.898 \\
\hline Error & 2.485 & 7 & 0.355 & & \\
\hline Total & 2561.551 & 11 & & & \\
\hline Corrected total & 16.462 & 10 & & & \\
\hline
\end{tabular}

In order to determine if there was a significant difference in fixation time between the four quadrants, and if the two factors had a significant effect on the fixation time, further analysis was needed.

Tab. 6 shows the results of a two-way ANOVA analysis of fixation time. This showed that the effect of interaction between the two factors on the fixation time was insignificant $(p>0.05)$. In addition, there was no significant correlation between shape-stable and the fixation time. Similarly, there was no significant correlation between decoration-disordered and the fixation time.

Tab. 6: 2-way ANOVA of fixation time.

\begin{tabular}{|l|c|c|c|c|c|}
\hline \multicolumn{1}{|c|}{ Source } & $\begin{array}{c}\text { type III Sum of } \\
\text { squares }\end{array}$ & df & Mean square & F & sig \\
\hline Shape-stable & 0.043 & 1 & 0.043 & 0.708 & 0.428 \\
\hline Decoration-disordered & 0.114 & 1 & 0.114 & 1.873 & 0.213 \\
\hline Shape-stable * decoration-disordered & 0.007 & 1 & 0.007 & 0.117 & 0.743 \\
\hline Error & 0.425 & 7 & 0.061 & & \\
\hline Total & 114.835 & 11 & & & \\
\hline Corrected total & 0.589 & 10 & & & \\
\hline
\end{tabular}




\section{DISCUSSION}

\section{Relationship between the eye movements and Kansei evaluation of Tang dynasty chairs.}

By using SD method and eye tracking system, the semantic space occupied by the Tang dynasty chairs could be divided into two main factors, which were shape and decoration. When the decoration of the chairs tended to be complicated, the number of fixation points increased, so the decoration had a significant correlation with the number of fixation points. Previous studies have indicated the relationship between people's attention and decoration (Zhang and Seo 2015). When the decoration is increased or complicated, people's attention and fixation points will focus on the decoration (Susac et al. 2019). This is because when people observe a product, if the decorative pattern is more complicated, people need to pay more attention to understand the relationship between the decorative pattern and other parts. When the decorative pattern of the chair is simple and harmonious, people's attention is mostly concentrated on the chair as a whole, and the fixation points are more concentrated (Hsu et al. 2017).

\section{Stimuli in the four quadrants}

Chairs in the shape-stable and decoration-disordered quadrant

The shape-stable and decoration-disordered quadrant included stimuli 1, 3, 9, and 10 (Fig. 8).

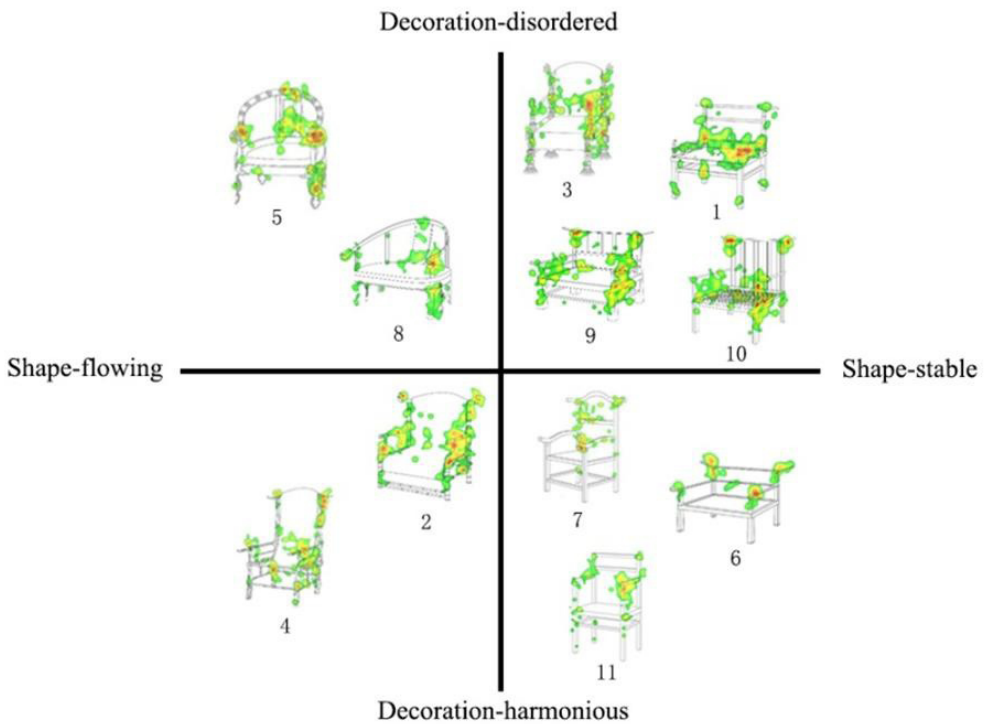

Fig. 8: Heat maps for stimuli in the quadrants of the Kansei space.

Stimulus 1 is the red-lacquered keyaki chair. This chair is a relic from the Imperial Household Agency. Stimulus 3 is the chair in Lu Luojia's painting "The Six Buddhas". Stimulus 9 is the chair in the "Zhen Yan Wu Zu" painting by Li Zhen from Tang dynasty. Stimulus 10 is the chair in the painting called "Eighteen Instruments of the Monk". Most of the chairs in this quadrant were evaluated as stable in shape and relatively complicated in decoration. Chairs in this quadrant were dominated by straight lines and stable forms. In previous studies, it has been 
found that smooth and stable straight lines generate a steady feeling in observers (Mata et al. 2017). The quantity of chair decoration in this quadrant is relatively high. The overall shape is complex. According to previous research (Hsu et al. 2017), when the decoration of chairs is too complicated, it will stimulate a subjective feeling of noise and disorder in observers.

\section{Chairs in the shape-stable and decoration-harmonious quadrant}

The shape-stable and decoration-harmonious quadrant included stimuli 6, 7 and 11. Stimulus 6 has the same origin as sample 9, but sample 6 is simpler in shape and has no excessive decorative components. Stimulus 7 is a chair in the mural of cave 9 in the Dunhuang Mogao Grottoes. Stimulus 11 is a chair in the mural of cave 148 in the Mogao Grottoes in Dunhuang. In stimuli 6, 7, and 11, the main shapes are straight lines, and the decoration is concentrated on the top of the backrest. The decoration of stimuli 6 and 7 is restricted to an upturned line on the upper side of the backrest, and the curved form of the armrest. The other components are simpler structural elements. Previous research shows that less-decorated products with simple lines and shapes (Du and Macdonald 2018) will generate both monotonous and harmonious subjective feelings in observers.

\section{Chairs in the shape-flowing and decoration-disordered quadrant}

The shape-flowing and decoration-disordered quadrant included stimuli 5 and 8. Stimulus 5 is a chair in the painting called "Emperor Taizong's Portrait". Stimulus 8 is a chair from the painting "Fair Lady", with a streamlined curve on the back of the chair. The decoration of stimuli 5 and 8 is more complicated, mainly due to the processes used to decorate the parts, including engraving and inlaying. The shape is streamlined, in the armrests, seat surfaces, and legs. As the study of Kapkin and Joines (2018), these smooth lines are considered feminine.

\section{Chairs in the shape-flowing and decoration-harmonious quadrant}

The shape-flowing and decoration-harmonious quadrant included stimuli 2 and 4 . Stimulus 2 is a chair in Lu Luojia's painting called "The Six Buddhas". Stimulus 4 is a chair in the painting called "Xiao Yi Cheated of the Orchid Pavilion". The shapes of stimuli 2 and 4 reflect the texture of the natural material. The top of the backrest, the armrest, and other parts have a curved shape. At the same time, the texture of the wood material itself reflects the fluidity. The decoration is reflected in the texture of the natural material. Research carried out by Song et al. (2016) shows that shapes based on natural materials give people a feeling of nature and harmony.

\section{Effect of Tang dynasty chair features on eye movements in Kansei evaluations}

Previous research (Nodine et al. 1993) has indicated that feature importance is correlated with a variety of gaze response data variables such as fixation time (Isham and Geng 2013), fixation points, and first-located time (Kubler et al. 2017). This gaze data provided insight into how people evaluate the features while making preference decisions (Du and Macdonald 2014, Ho and $\mathrm{Lu}$ 2014, Husic-Mehmedovic et al. 2017). In this study, the combination of subjective evaluation and eye-movement tracking meant that people's gazes could be more accurately and rapidly understood (Du and Macdonald 2015), so that responses to the morphological characteristics of Tang dynasty chairs could be assessed and understood. Hsu et al. (2017) concluded that the seats and backrests were the two most important features of chair design; however, the results of the current experiment are inconsistent with this conclusion. According to the heat maps produced in this experiment, backrest, armrests, and legs were all important features of the Tang dynasty chairs (Fig. 9). 


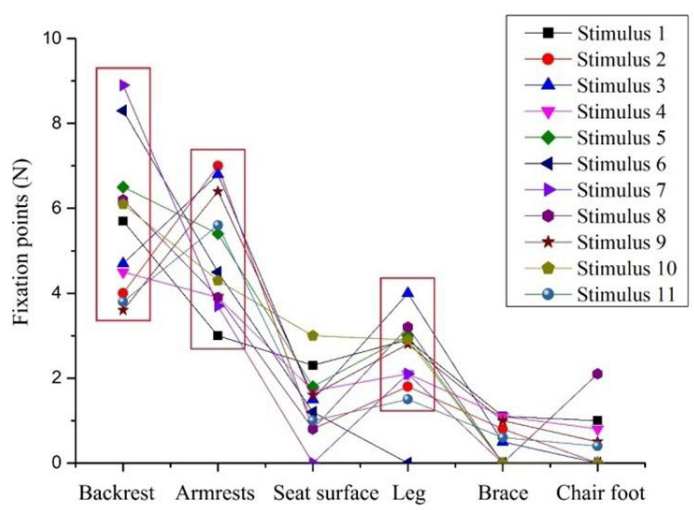

Fig. 9: Fixation points of stimuli's different parts.

This indicates that the subjects considered these all to be important features of the overall chair forms. These results can be used to guide modern designs based on these ancient precursors. As summarized by the study of Wan et al. (2018), subjects paid more attention to the decorative details on the chairs, implying that appropriate design and decoration may improve people's levels of interest in the chairs. When designing a Tang dynasty style chair, designers can, therefore, focus on the backrest, armrests, and legs of the chairs, in accordance with the observed cognitive responses to the Tang dynasty chairs. Through the careful design of the morphological characteristics of modern Tang dynasty style chairs, it will be possible to accurately recreate the style of the Tang dynasty chairs on the basis of the ancient designs characteristics.

The decoration of Tang dynasty chairs can be complicated, but it is mostly concentrated on the upper side of the backrest, on the armrests, and on the legs. In the future, designers attempting to create modern chairs based on Tang dynasty style chair designs should, therefore, focus on all three important areas of the Tang dynasty chairs.

\section{CONCLUSIONS}

The main conclusions of this study are as follows: (1) Through SD evaluation method, it was shown that the two factors that affected the Kansei evaluations were shape and decoration. The complex and harmonious levels of decoration had significant impact on participants' fixation points. (2) By combining Kansei evaluations and eye tracking, it was found that the main features that affect people's evaluation of the Tang dynasty chairs are backrests, armrests, and legs.

This finding demonstrated that designers need to consider these three main features when designing Tang dynasty style furniture. By combining Kansei evaluations with eye tracking, the eye movements associated with Tang dynasty chairs can be observed, so that the Tang dynasty furniture features hat affect people's perceptual cognition can be quickly and accurately analyzed. Significant features identified in the experiment can be researched in more detail, new chairs can be designed with an emphasis on these details, and the discovery in this experiment can also be used to predict people's perceptual evaluations of new designs. The methods used and the conclusions drawn in this study can be provided to designers, engineers, and related researchers to guide future designs of Tang dynasty style chairs. 


\section{ACKNOWLEDGMENT}

This work was supported bya Project Funded by the National First-class Disciplines (PNFD), and A Priority Academic Program Development of Jiangsu Higher Education Institutions (PAPD).

\section{REFERENCES}

1. Castilla, N., Llinares, C., Bravo, J.M., Blanca, V., 2017: Subjective assessment of university classroom environment. Building and environment 122: 72-81.

2. Dong, M.Y., Zhou, H.B., Jiang, X.M., Lu, Y., Wang, W.B., Yin, Y.F., 2017: Wood used in ancient timber architecture in Shanxi Province, China. IAWA Journal 38(2): 182-200.

3. Du, P., Macdonald, E.F., 2014: Eye-tracking data predict importance of product features and saliency of size change. Journal of Mechanical Design 136(8): 081005.

4. Du, P., Macdonald, E.F., 2015: Products' shared visual features do not cancel in consumer decisions. Journal of Mechanical Design 137(7): 071409.

5. Du, P., Macdonald, E.F., 2018: A test of the rapid formation of design cues for product body shapes and features. Journal of Mechanical Design 140(7): 071102.

6. Guo, F., Ding, Y., Liu, W.L., Liu, C., Zhang, X.F., 2016: Can eye-tracking data be measured to assess product design? : Visual attention mechanism should be considered. International Journal of Industrial Ergonomics 53: 229-235.

7. Ho, C.H., Lu, Y.N., 2014: Can pupil size be measured to assess design products? International Journal of Industrial Ergonomics 44(3): 436-441.

8. Hsu, C.C., Fann, S.C., Chuang, M.C., 2017: Relationship between eye fixation patterns and Kansei evaluation of 3D chair forms. Displays 50: 21-34.

9. Hu, W.G., Liu, N., Guan, H.Y., 2019b: Experimental study of the contact forces and deformations of mortise-and-tenonjoints considering the fits and grain orientations of the tenon. Bioresources 14(4): 8728-8737.

10. Hu, W.G., Liu, N., Guan, H.Y.,2019c: Optimal design of a furniture frame by reducing the volume of wood. Drewno 62(204): 85-97.

11. Hu, W.G., Wan, H., Guan, H.Y.,2019a: Size effect on the elastic mechanical properties of beech and its application in finite element analysis of wood structures. Forests 10(9): 783.

12. Husic-Mehmedovic, M., Omeragic, I., Batagelj, Z., Kolar, T., 2017: Seeing is not necessarily liking: Advancing research on package design with eye-tracking. Journal of business research 80: 145-154.

13. Isham, E.A., Geng, J.J., 2013: Looking time predicts choice but not aesthetic value. Plos One 8(8): e71698.

14. Kapkin, E., Joines, S., 2018: An investigation into the relationship between product form and perceived meanings. International Journal of Industrial Ergonomics 67: 259-273.

15. Khalighy, S., Green, G., Scheepers, C., Whittet, C., 2015: Quantifying the qualities of aesthetics in product design using eye-tracking technology. International Journal of Industrial Ergonomics 49: 31-43.

16. Kubler, T.C., Rothe, C., Schiefer, U., Rosenstiel, W., Kasneci, E., 2017: SubsMatch 2.0: Scanpath comparison and classification based on subsequence frequencies. Behavior Research Methods 49(3): 1048-1064. 
17. Lee, J., Kong, Y.H., Luo, M., 2018: Syntactic patterns in classical Chinese poems: A quantitative study. Digital Scholarship in the Humanities 33(1): 82-95.

18. Loyola, P., Martinez, G., Munoz, K., Velasquez, J.D., Maldonado, P., Couve, A., 2015: Combining eye tracking and pupillary dilation analysis to identify website key objects. Neurocomputing 168: 179-189.

19. Mata, P.M., Ahmed-Kristensen, S., Brockhoff, P.B., Yanagisawa, H., 2017: Investigating the influence of product perception and geometric features. Research in Engineering Design 28(3): 357-379.

20. Nodine, C.F., Locher, P.J., Krupinski, E.A., 1993: The role of formal art training on perception and aesthetic judgment of art compositions. Leonardo 26(3): 219-227.

21. Song, S.S., Wan, Q. Wang, G.G., 2016: Eye movement evaluation of different wood interior decoration space. Wood Research 61(5): 831-843.

22. Susac, A., Bubic, A., Planinic, M., Movre, M., Palmovic, M., 2019: Role of diagrams in problem solving: An evaluation of eye-tracking parameters as a measure of visual attention. Physical Review Physics Education Research 15(1): 013101.

23. Trujillo, J.L.H., Avino, A.M.I., Millan, C.L., 2016: User evaluation of neonatology ward design: An application of focus group and semantic differential. Herd-Health Environments Research \& Design Journal 10(2): 23-48.

24. Vieira, J., Osorio, J.M.A, Mouta, S., Delgado, P., Portinha, A., Meireles, J.F., Santos, J.A., 2017: Kansei engineering as a tool for the design of in-vehicle rubber keypads. Applied Ergonomics. 61: 1-11.

25. Wan, Q. Song, S.S., Li, X.H., Zhang, Q. Yang, X., Zhang, Y.C., Fei, B.H., Yao, L.H., 2018: The visual perception of the cardboard product using eye-tracking technology. Wood Research 63(1): 165-178.

26. Wan, Q., Wang, G.G., Zhang, Y.C., Song, S.S., Fei, B.H., Li, X.H., 2018: Cognitive processing toward traditional and new Chinese style furniture: evidence from eye-tracking technology. Wood research 63(4): 727-739.

27. Wang, C.C., Yang, C.H., Wang, C.S., Chang, T.R., Yang, K.J., 2016: Feature recognition and shape design in sneakers. Computer \& Industrial Engineering 102: 408-422.

28. Wang, Y.H., Zhang, H.H., Zhao, Y.N., Hao, W., Ning, X.J., Shi, Z.H., Zhao, M.H., 2015: Three-dimensional reconstruction method of Tang dynasty building based on point clouds. Optical Engineering 54(12): 123111.

29. Winston, A.S., Cupchik, G.C., 1992: The evaluation of high art and popular art by naive and experienced viewers. Visual Arts Research 18(1): 1-14.

30. Ying, W., 2018: A national flower's symbolic value during the Tang and Song dynasties in China. Space and Culture 21(1): 46-59.

31. Zanous, H.P., Sangari, E., 2018: The last sasanians in Chinese literary sources: recently identified statue head of a sasanian prince at the Qianling Mausoleum. Iranian Studies 51(4): 499-515.

32. Zhang, B.Y., Seo, H.S., 2015: Visual attention toward food-item images can vary as a function of background saliency and culture: An eye-tracking study. Food Quality and Preference 41: 172-179.

33. Zhou, J., Guo, G., Dong, F.Y., Li, H., Lin, L., Yang, F., 2014: Multi-dimensional method for evaluating a product's conceptual schemes. South African Journal of Industrial Engineering 25(3): 184-198.

34. Zhu, Y., 2016: Different cultures of computation in seventh century China from the viewpoint of square root extraction. Historia Mathematica 43(1): 3-25. 
Zhonghua $Z_{\text {hagn, Boming }} \mathrm{XU}^{*}$

College of Furnishings and Industrial Design

Nanjing Forestry University

NANJING 210037

China

*Corresponding author: xubomingnjfu@126.com

Phone: +8615568370432 